\title{
Cerebral blood velocity in subarachnoid haemorrhage: a transcranial Doppler study
}

\author{
JEFFREY S COMPTON, SHEILA REDMOND, LINDSAY SYMON \\ From the Gough Cooper Department of Neurosurgery, Institute of Neurology, London, UK
}

SUMMARY A study examining the utility of transcranial Doppler ultrasound for the determination of cerebral vasospasm following subarachnoid haemorrhage is reported. A control group of 21 patients and a second group of 20 patients suffering from subarachnoid haemorrhage or aneurysm and a group of 26 with other intracranial pathologies were studied. The Doppler flow velocity (DFV) was significantly higher when vasospasm was present. If it was higher than $100 \mathrm{~cm} / \mathrm{s}$, the patients were found to have vasospasm in $80 \%$ of cases. If Doppler flow velocity was below 100 , less than $10 \%$ had spasm. Doppler flow velocity was not found to increase following craniotomy in patients not suffering from subarachnoid haemorrhage. In subarachnoid haemorrhage patients there was a trend to increased Doppler flow velocity especially in patients who developed neurological deficit. Doppler flow velocity and Initial Slope Index by xenon clearance (ISI) were not found to correlate with clinical grade. The ISI/DFV quotient (which can be shown mathematically to be related to vessel diameter) was found to correlate well with clinical grade. In this largely post-operative group, absolute levels, or rate of change of Doppler flow velocity could not be shown to be related to the onset or existence of neurological deficit.

Since radiologically identifiable spasm of the major intracranial arteries following subarachnoid haemorrhage (SAH) was first described by Ecker and Riemenschneider in $1915,{ }^{1}$ controversy has surrounded its relationship to neurological state, prognosis and its effect on investigation and treatment. Little doubt now remains, however, that the presence of vasospasm relates to grade and outcome. ${ }^{23}$ It has also been demonstrated that vasospasm can occur within 24 hours of haemorrhage and last for 1 to 4 weeks. ${ }^{4}$

It is also true that a patient's neurological state can deteriorate because of vasospasm. The problem of elucidating the cause of any such deterioration and distinguishing vasospasm from rebleeding, hydrocephalus, oedema following cerebral retraction during operation or infection still taxes the surgeon. Whilst it was hoped that with the development of non-invasive methods for measuring cerebral blood flow, the diagnosis of vasospasm would be simplified, these methods remain insensitive and at best poorly

Address for reprint requests: Professor L Symon, National Hospital, Queen Square, London WC1N 3BG, UK.

Received 20 June 1986 and in final revised form 8 April 1987. Accepted 16 April 1987 discriminative. Cerebral angiography remains the "gold standard" for the demonstration of vasospasm.

With the modification of Doppler ultrasound by Aaslid $^{5}$ to enable the transcranial measurement of the velocity of cerebral blood flow, the feasibility of using such a method to demonstrate the presence of vasospasm has been expounded.

The authors have undertaken a study to examine the relationship between the cerebral blood velocity, appearance of the cerebral vasculature on angiogram, cerebral blood flow, and the patients' clinical condition and progress, in an effort to determine its efficacy in confirming or refuting the presence of vasospasm.

\section{Clinical material and methods}

Three groups of patients were studied. A group of patients without intracranial pathology were studied to determine normal values. Patients suffering subarachnoid haemorrhage or aneurysm comprised the second group (subarachnoid haemorrhage from demonstrated arteriovenous malformation was excluded as the blood flow within the malformation caused spurious blood velocity values). A third group, comprised patients undergoing craniotomy for a variety of pathologies with which the subarachnoid haemorrhage group could be compared. This was done in an effort to see the effect of craniotomy for non-vascular pathology on the cerebral blood flow velocity.

The age and sex distribution of the patients studied is 
shown in table 1. There was no significant difference in the average age of the patients in each of the groups. Three patients in each of the control and subarachnoid haemorrhage groups were hypertensive. None of the patients in the "other pathologies" group were hypertensive. Table 2 lists the pathological conditions which comprised the "other pathologies" group.

Table 3 details the subarachnoid haemorrhage group. Subarachnoid patients presented from 1 to 21 days post haemorrhage. On presentation 10 were Hunt and Hess grade I, four were grade II, one grade III, and five grade IV. Three patients were hypertensive (diastolic BP greater than $90 \mathrm{~mm}$ $\mathrm{Hg}$ or systolic BP more than $160 \mathrm{~mm} \mathrm{Hg}$ ) at some stage during their illness.

All patients had measurement of the blood velocity in the anterior circulation vessels, by the Doppler method described by Aaslid, ${ }^{5}$ using an EME TC2-64 transcranial Doppler (with a standard $2 \mathrm{MHz}$ probe). Measurements of the Doppler flow velocity (DFV), using the time averaged mean Doppler flow velocity were recorded for the anterior cerebral, internal carotid (ICA), and middle cerebral arteries, routinely. The method described by Aaslid for confirming the vessel studied was used. Briefly, with the probe depth set to 50 or $55 \mathrm{~mm}$ a vessel was located by varying the angle of the probe, starting just above the zygoma. Once a vessel was located, it was followed peripherally (by decreasing the gated depth) until the presence of flow to and from the probe at about $30 \mathrm{~mm}$ confirmed it to be the middle cerebral. This vessel was then followed medially until the bifurcation of the internal carotid was identified, again by the location of to and from flow. It is well known that errors in assessing Doppler flow velocity will increase with in-

Table 1 Patient data

\begin{tabular}{llll}
\hline & Control & $S A H^{*}$ & Other pathologies \\
\hline No & 21 & 20 & 26 \\
M/F & $9 / 12$ & $6 / 14$ & $14 / 12$ \\
Age (yr) & $53 \cdot 09 \pm 19 \cdot 1$ & $45 \cdot 2 \pm 10 \cdot 7$ & $49 \cdot 56 \pm 32 \cdot 3$ \\
(mean \pm SD) & & & \\
\hline
\end{tabular}

*SAH includes all aneurysm, and SAH patients.

Table 2 Pathological conditions in "Other Pathologies" group

\begin{tabular}{lrr}
\hline Condition & Number \\
\hline Tumour & 13 & \\
Supratentorial & & 3 \\
$\quad$ Meningioma & 4 \\
$\quad$ Gliomata & 1 \\
$\quad$ Metastases & & 2 \\
Subtentorial & & 2 \\
$\quad$ Accoustic & & 1 \\
$\quad$ Gliomata & 6 & 1 \\
$\quad$ Metastasis & & 4 \\
Trauma & & 1 \\
$\quad$ Extradural & 7 & 2 \\
Subdural (ac/ch) & & 4 \\
Diffuse Axonal Injury & & 1 \\
Others & 26 & \\
Intracerebral Haemorrhage & & \\
$\quad$ Hydrocephalus & & \\
Cerebral abscess & &
\end{tabular}

Table 3 Patients treated for $S A H$ or aneurysm

\begin{tabular}{lll}
\hline & SAH & Unruptured Aneurysm \\
\hline Anterior Comm & 6 & 1 \\
Middle Cereb & 3 & 2 \\
Carotid & 3 & 1 \\
Unknown* & 4 & - \\
\hline
\end{tabular}

*2 died before angiography, 2 cases with normal angiography.

creasing angle of incidence of the insonnating beam. In this study the internal carotid flow at the level of bifurcation was recorded. Absolute values of ICA velocity are no doubt unreliable, but comparisons from day to day and ICA to ICA may well be more useful, as the angle of insonnation is unlikely to change drastically. The Al could then be followed medially, by further increasing the depth of sampling.

All groups were studied before operation. The subarachnoid haemorrhage and "other pathology" groups were also studied after operation, usually twice daily to establish the time course of the Doppler flow velocity following operation. The subarachnoid haemorrhage patients also had regional cerebral blood flow (rCBF) measured using a Novo ${ }^{R}$ 2a Cerebrograph, employing the $\mathrm{Xe}^{133}$ clearance method. ${ }^{8}$ CBF was usually assessed on admission and three to five times post-operatively (6 hours post-operatively, 1 and 3 days post-operatively and with other studies depending on clinical state). The Initial Slope Index (ISI) was studied, as the parameter felt to be most relevant in the pathological state.

Subarachnoid haemorrhage patients were treated along standard clinical lines. Subarachnoid haemorrhage was confirmed by CT scanning or lumbar puncture. The patients' clinical condition was quantified according to the grading schema proposed by Hunt and Hess. ${ }^{6}$ Patients in grade III or better had angiography soon after admission. All patients received dexamethasone, an anticonvulsant, and were maintained on a regimen of bed rest. Operation was performed regardless of time since haemorrhage, when the patient was found to be grade I or II. This policy generally resulted in a relatively early operation.

\section{Results}

The authors found little difficulty in estimating the Doppler flow velocity in most patients. With practise, an uncomplicated study could be performed in 15 to 20 minutes. All but the most violent patients could be studied. In some cases, talking by the patient caused interference, and in some postoperative cases the bulk of the head dressing or pain from the wound caused minor difficulty, but prevented study in only two instances.

\section{Normal values}

Doppler flow velocity on admission is shown in table 4. The results were also analysed in relation to the incidence of Doppler flow velocity above $100 \mathrm{~cm} / \mathrm{s}$. In the control group Doppler flow velocity exceeded $100 \mathrm{~cm} / \mathrm{s}$ in one of $42(2 \cdot 38 \%)$ hemispheres studied. In 
Table 4 Pre-operative $D F V($ Mean $\pm S D(N))$

\begin{tabular}{llll}
\hline & Control & $S A H^{*}$ & Other pathologies \\
\hline AC & $37.63 \pm 10.63(38)$ & $34 \cdot 38 \pm 14.97(26)$ & $37 \cdot 00 \pm 12 \cdot 15(23)$ \\
MCA & $50 \cdot 84 \pm 32.60(38)$ & $45 \cdot 71 \pm 27.95(26)$ & $50 \cdot 25 \pm 20 \cdot 18(31)$ \\
ICA & $42.73 \pm 9.94(40)$ & $41 \cdot 38 \pm 14.77(26)$ & $42.30 \pm 14.22(30)$ \\
\hline
\end{tabular}

*DFV on admission

the other pathologies" group there were nine instances of $322(2 \cdot 8 \%)$ hemisphere estimations where the Doppler flow velocity exceeded $100 \mathrm{~cm} / \mathrm{s}$ (Chi square $=12$; NS). in the subarachnoid haemorrhage group, 102 of 364 had Doppler flow velocity greater than $100 \mathrm{~cm} / \mathrm{s}$ (Chi square compared with control group $=13.07 ; p<0.001$; compared with "other pathologies" group Chi square $=85.0 ; \mathrm{p}<0.001$ ).

\section{Doppler flow velocity and angiography}

An attempt was made to compare the Doppler flow velocity obtained immediately pre-angiogram, with the diameters of the vessels shown on the angiogram. The angiograms were classified as to whether spasm was present or not (by an independent observer, unaware of the patients' clinical state or the Doppler flow velocity results). The presence or absence of spasm was noted and its location, severity and extent recorded. These results were correlated with the presence of high Doppler flow velocity results (Doppler flow velocity greater than $100 \mathrm{~cm} / \mathrm{s}$ for any portion of the readings of the appropriate hemisphere).

It was found that a higher proportion of vessels demonstrating angiographic spasm had Doppler flow velocity above $100 \mathrm{~cm} / \mathrm{s}$ (no spasm; 1/24 had Doppler flow velocity above $100 \mathrm{Cf}$. spasm present $4 / 6$ had Doppler flow velocity above 100 ; Chi square $=9 \cdot 37$; $\mathrm{p}<0.01)$. The mean of the highest Doppler flow velocity in the middle cerebral arteries which showed spasm was significantly higher than that of the nonspastic middle cerebral vessels (spasm present, mean Highest, Doppler flow velocity $=93.33 \pm 31.44(6)$; no spasm $=52 \cdot 0 \pm 21 \cdot 17(24) ; \mathrm{t}=2 \cdot 94, \mathrm{p}<0.05)$.

\section{Doppler flow velocity and craniotomy}

Doppler flow velocity was found not to increase following craniotomy in the "other pathology" group of patients. Likewise the proportion of estimations above $100 \mathrm{~cm} / \mathrm{s}$ remained at $2 \cdot 5 \%$.

Figure 1 presents the time course of Doppler flow velocity for both the "other pathology" group and the subarachnoid haemorrhage group of patients. There is a trend for Doppler flow velocity to increase in the subarachnoid haemorrhage group, following craniotomy, especially in the group of patients which developed new deficit. The wide scatter of the data prevents statistical demonstration of significance in this small group of patients.

\section{Correlation with clinical condition}

Figure 2 presents the graph of Doppler flow velocity vs clinical grade according to Hunt and Hess. The highest Doppler flow velocity of each study is used for calculation of the mean, and has been plotted, as it would seem logical that it is the highest flow velocity (representing the most spastic artery) which determines the clinical status. No significant relationship is demonstrated, although a trend for Doppler flow velocity to increase in grade III is evident. Likewise in the data presented in fig 3 , whilst showing the tendency of ISI to decrease with worsening clinical grade, there is no significant difference, between one grade and the next.

A better relationship was however found, using a combination of Doppler flow velocity and ISI, and is shown in fig 4. The radius ratio (ISI/DFV) significantly falls from grade I to II and then from II to III and III to IV ( $p<0.01$ for each).

Aaslid and colleagues ${ }^{7}$ have reported features in the Doppler flow velocity which have, in their hands been predictive of neurological deficit. Their group found that Doppler flow velocity greater than $200 \mathrm{~cm} / \mathrm{s}$ was associated with a tendency for ischaemia, that flow velocity between 120 and $140 \mathrm{~cm} / \mathrm{s}$ never led to brain infarction, and that a rapid rise in Doppler flow velocity to levels above $200 \mathrm{~cm} / \mathrm{s}$ was a particularly bad portent.

In the present study eight of 20 patients developed new deficit in the course of their illness, which CT proved was not due to hydrocephalus or rebleeding. Two patients awoke, post-operatively with new deficit, whist five developed it 2 to 5 days after operation, and one before operation. In four cases the deficit persisted. In only three of the eight cases did the Doppler flow velocity exceed $200 \mathrm{~cm} / \mathrm{s}$, and in no case did it rise before the onset of symptoms. In one of these cases the symptoms proved transient. Furthermore, in three of 12 patients who suffered no new neurological sequelae, Doppler flow velocity rose rapidly (within 12 hours) to values above $200 \mathrm{~cm} / \mathrm{s}$.

\section{Discussion}

The authors have confirmed the ease with which the flow velocity of blood in the basal vessels of the anterior circulation can be insonnated. The correlation of increased velocity of flow with the angiographic appearance of vasospasm is made, such that patients who have pre-angiography DFVs greater than 100 $\mathrm{cm} / \mathrm{s}$ are highly likely to have angiographic spasm. If DFV exceeds $100 \mathrm{~cm} / \mathrm{s}$ there is a $80 \%$ chance that the angiogram will reveal vasospasm. If DFV is less than 100 , there is less than a $10 \%$ chance that vasospasm will be present. It is notable that no correlation be- 

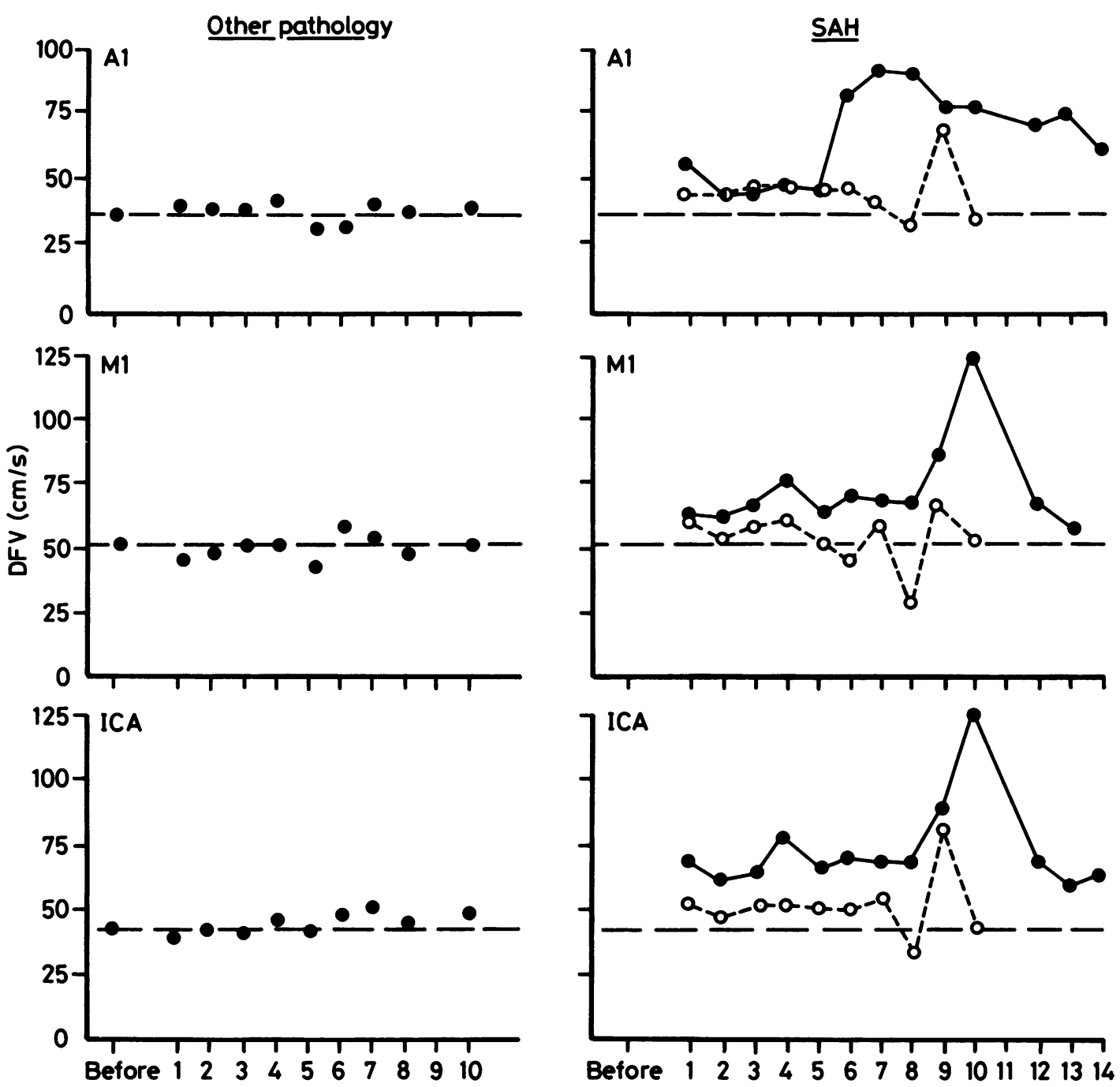

After operation (days)

Fig 1 Mean DFV before and after craniotomy. Plot of mean DFV plotted against time. Left panels, other pathology group. Right panels SAH group showing those patients which developed deficit (O-O) and no new deficit ( $\bigcirc-\bigcirc)$. Upper panels, anterior cerebral, middle, middle cerebral, lower, internal carotid. The dashed line is the control value for each vessel.

tween the DFV and the severity of vasospasm could be deduced in this small number of cases.

It is also likely that the early time following SAH at which angiography was performed coincides with the early period which is known to have a relatively low incidence of spasm.

In short, transcranial Doppler ultrasonography is shown to be a non invasive method of predicting the presence of angiographic vasospasm. Aaslid and coworkers have pointed out the theoretical problems associated with angular malalignment of the probe.

In a recently reported study, Aaslid and colleagues ${ }^{7}$ have reported features in the DFV which have, in their hands been predictive of neurological deficit. Although this is only a small number of patients, the authors feel that the results demonstrate that in the post-operative patient, the absolute value of DFV loses its diagnostic value of ischaemic neurological deficit. In any individual, high $(200 \mathrm{~cm} / \mathrm{s})$ or rapid rise in DFV is not necessarily of alarming significance, nor 


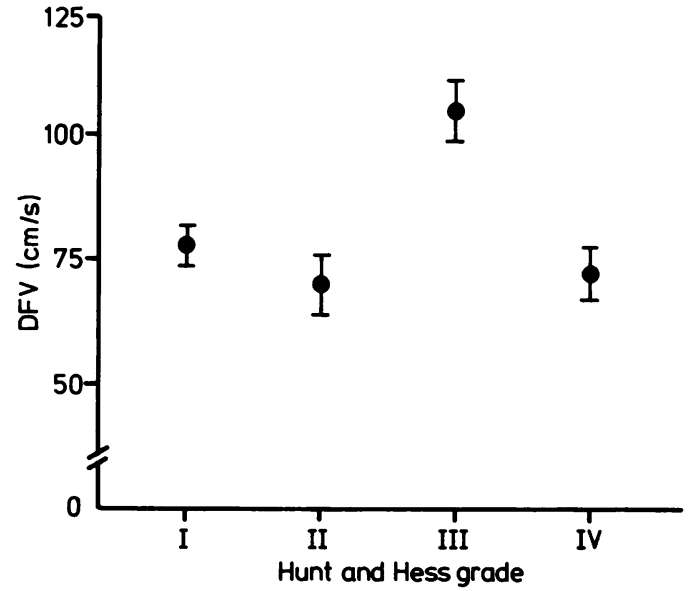

Fig 2 Mean Highest DFV vs Hunt and Hess grade at the time of study. (Mean $\pm S E M)$.

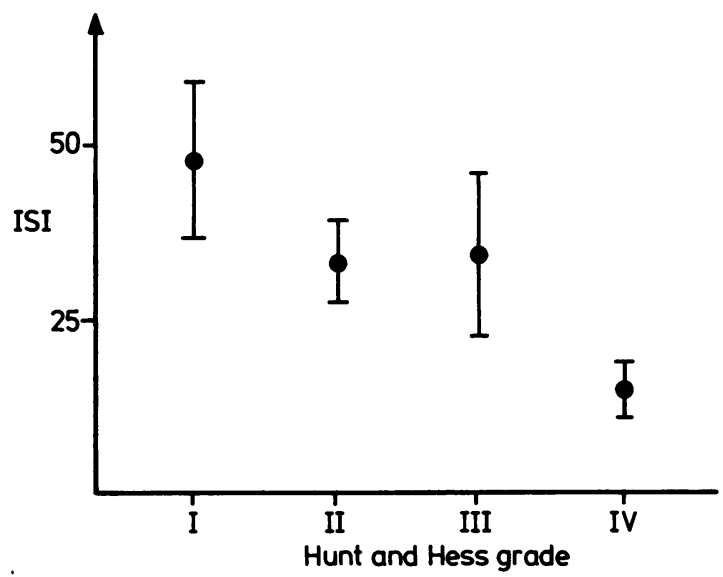

Fig 3 Mean ISI vs Hunt and Hess grade at the time of study. (Mean $\pm S D)$.

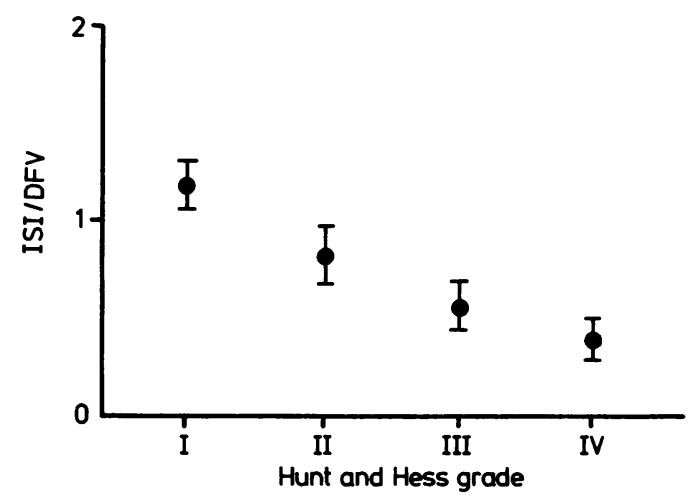

Fig 4 Radius Ratio (ISI/DFV) vs $H$ and $H$ grade at the time of study. (Mean $\pm S D)$. is low DFV of significant reassurance.

The present study does demonstrate that in the normal course of events, craniotomy in its own right does not increase the DFV. DFV does tend to increase following craniotomy for SAH, perhaps suggesting the increased irritability of the cerebral vasculature following haemorrhage. Higher DFVs do tend to occur in patients who develop neurological deficit, but the wide variation of the DFV makes statistical interpretation difficult.

The lack of correlation between DFV and clinical grade deserves comment. The authors propose that the normal response by the brain to ischaemia due to vasospasm is to maintain the CBF at or near normal levels, and that this is achieved by increasing the blood flow velocity. A situation therefore exists where although the CBF and therefore clinical state are normal, the DFV is increased. It is also obvious that there is more to the determination of clinical condition than blood flow alone.

Also of interest is the relationship between the ISI and DFV. Gilsbach ${ }^{9}$ amongst others has pointed out the relationship between flow and velocity, viz that $\mathrm{v}=$ flow $/\left(\right.$ pi. $\left.\mathrm{r}^{2}\right)$ or $\mathrm{r}^{2}=$ flow $/($ pi.v). The authors have shown that this relationship, using the ISI as the flow term, corresponds best with the patients' clinical condition.

\section{References}

1 Ecker A, Riemenschneider PA. Arteriographic demonstration of spasm of the intracranial arteries with special reference to saccular arterial aneurysms. $J$ Neurosurg 1951;8:660-7.

2 Weir B, Grace M, Hansen J, et al. Time course of vasospasm in man. $J$ Neurosurg 1978;48:173-8.

3 Loach AB, De Azevedo-Filho HRC. Some observations in the microeneurosurgical treatment of intracranial aneurysms. Acta Neurochim 1976;35:97-103.

4 du Boulay G. Distribution of spasm in the intracranial arteries after subarachnoid haemorrhage. Acta Radiol Diag 1963;1:257-66.

5 Aaslid R, Huber P, Nornes H. Evaluation of cerebrovascular spasm with transcranial Doppler ultrasound. J Neurosurg 1984;60:37-41.

6 Hunt WE, Hess RM. Surgical risk as related to time of intervention in the repair of intracranial aneurysm. $J$ Neurosurg 1968;28:14-19.

7 Seiler RW, Grolimund P, Aaslid R, et al. Cerebral vasospasm evaluated by transcranial ultrasound correlated with clinical grade and CT-visualized subarachnoid hemorrhage. J Neurosurg 1968;64:594-9.

8 Rosenstein J, Suzuki M, Symon L, et al. Clinical use of a portable bedside cerebral blood flow machine in the management of aneurysmal subarachnoid haemorrhage. $r C B F$ Bulletin 1986;9:183-90.

9 Gilsbach JM. Intraoperative Doppler Sonography in Neurosurgery. Springer Verlag/Wein. 1983. 